\title{
Les médias africains à l'heure du numérique, Annie Lenoble-Bart et Annie Chéneau-Loquay (dir.)
}

L'Harmattan, Netsuds, n 5, septembre 2010, 133 p. ISBN

978-2-296-12584-1

\section{Alain Kiyindou}

\section{(2) OpenEdition}

\section{Journals}

Édition électronique

URL : http://journals.openedition.org/communicationorganisation/1339

DOI : 10.4000/communicationorganisation. 1339

ISSN : $1775-3546$

Éditeur

Presses universitaires de Bordeaux

Édition imprimée

Date de publication : 1 juin 2010

Pagination : 183-184

ISBN : 978-2-86781-710-6

ISSN : $1168-5549$

\section{Référence électronique}

Alain Kiyindou, "Les médias africains à l'heure du numérique, Annie Lenoble-Bart et Annie ChéneauLoquay (dir.) ", Communication et organisation [En ligne], 37 | 2010, mis en ligne le 31 mai 2011, consulté le 21 septembre 2020. URL : http://journals.openedition.org/communicationorganisation/ 1339 ; DOI : https://doi.org/10.4000/communicationorganisation.1339

Ce document a été généré automatiquement le 21 septembre 2020.

(C) Presses universitaires de Bordeaux 


\section{Les médias africains à l'heure du numérique, Annie Lenoble-Bart et Annie Chéneau-Loquay (dir.)}

L'Harmattan, Netsuds, $n^{\circ}$ 5, septembre 2010, 133 p. ISBN

978-2-296-12584-1

\section{Alain Kiyindou}

1 Annie Lenoble-Bart et Annie Chéneau-Loquay présentent dans ce cinquième numéro des cahiers de Netsuds (CEAN-CNRS/Africanti), sept articles traitant des conditions et formes d'usages des Technologies de l'information et de la communication dans les médias africains. Dans l'introduction, Annie Lenoble-Bart souligne le lien avec les défis et perspectives du développement par le biais de la focale médiatique. Le numéro reste ancré principalement dans les travaux du Groupe de recherche sur les médias africains de l'Équipe d'Accueil MICA de l'Université de Bordeaux, tout en précisant que les «nouvelles» technologies de l'information et de la communication renouvellent un certain nombre de questions. La palette des objets est variée, elle va du fax à l'internet en passant par la presse écrite et la radio.

2 D'entrée de jeu, les auteurs tiennent à placer la démocratisation de la presse à la suite de longues batailles menées depuis de nombreuses années comme pour se départir du lien arbitraire souvent établi entre journalisme en ligne et démocratisation de la presse. L'exemple du Mozambique retrace ce cheminement dans lequel les moyens de communication «anciens » comme le fax ont aussi joué leur rôle. Comme le rappelle Claudio Jone, le combat pour la liberté d'expression dans ce pays fut rude du fait de la confiscation de la liberté par le Frelimo, parti au pouvoir. Le Sms (Short message service), de plus en plus utilisé par les journalistes africains vient donc en prolongement du fax et autres moyens de communication à distance. C'est d'ailleurs cette pratique que décrit Norbert Ouendji. En effet, explique-t-il, l'usage des Sms dépasse le cadre privé et est de plus en plus manifeste dans la sphère journalistique où l'urgence est une exigence absolue. La téléphonie mobile permet, dans sa légèreté et sa praticité, de palier les difficultés liées aux systèmes classiques de transport de données. S'appuyant 
sur une enquête de terrain, Cyriaque Paré tente d'étudier l'appropriation des technologies de l'information et de la communication par les médias africains. Ce travail fondé essentiellement sur l'analyse des sites internet n'aborde pas directement les questions liées à la pratique des TIC par les journalistes. Tout se passe comme s'il y avait un modèle type de site internet et l'auteur tente, à partir de son analyse, de déceler les écarts entre le site «idéal » et les sites des médias africains en se basant notamment sur des considérations ergonomiques. Une telle approche laisse donc de côté les inventions et tout autre "art de faire " africain. La question des médias est aussi analysée en lien avec la diaspora africaine. Les sites portails étudiés par Moda Gueye laissent apparaître une prédominance de débats sur la vie politique conférant au cyberespace l'aspect d'un véritable espace public où se forgent des opinions, avec des leaders, des personnes visibles, audibles et d'autres qui le sont moins. En tous cas, l'investissement dans la production et l'animation des sites internet sénégalais semble déterminer leur vitalité. Mais au-delà des sites de la diaspora, la presse en ligne offre également de véritables espaces de débats analysés ici par Sidiki Bamba. Sur Abidjannet par exemple, les internautes sont appelés à donner leur avis sur les articles publiés et à interpeller l'auteur qui à son tour peut leur répondre. Mais la question sous-jacente est celle de savoir si Internet facilite ou refonde le débat public.

3 La radio, un des médias le plus utilisé, est aussi exposée aux nombreuses mutations dues aux avancées technologiques et confirme encore plus son rôle de média citoyen. Etienne Damome explique qu'il existe aujourd'hui une multitude de radios qui n'ont d'existence qu'en ligne. Exploitant à fond le jeu de l'interactivité, elles sont de véritables espaces de dialogue avec les auditeurs et se démarquent grâce à la personnalisation des programmes, des contenus encore plus denses, mais internet, précise l'auteur, ne semble pas avoir réglé tous les défis. Il n'a pas amélioré la qualité des programmes et augmenté la production. Mais l'engouement pour la radio en ligne contraste avec le contexte de diffusion et risque d'avoir pour conséquence la marginalisation de certaines populations. Ces bouleversements posent à l'évidence la question de la formation du journaliste aux nouvelles pratiques de son métier. Au CESTI, cet apprentissage passe par le "Dakar blog ", dispositif de formation que décrit Mamadou Ndiaye. Mais si l'article part d'un cas concret, il passe à côté des problèmes réels posés par la formation à un métier en pleine mutation.

4 En définitive, le propos développé dans ce numéro concerne les médias africains confrontés au déploiement des technologies de l'information et de la communication dans une approche critique intégrant toute la complexité dans laquelle se déploient aussi bien les médias que les "nouvelles" technologies de l'information et de la communication, une complexité qui amène à repenser les usages, les contenus mais aussi les pratiques journalistiques. 